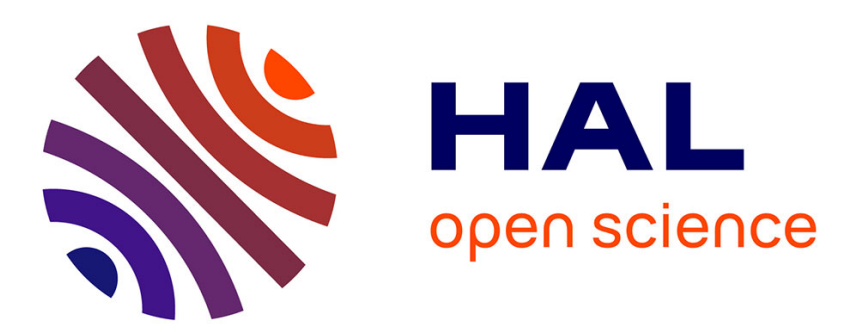

\title{
The art of scaling up : a computational account on action selection in basal ganglia
}

Bhargav Teja Nallapu, Nicolas P. Rougier, Bapi Raju Surampudi

\section{To cite this version:}

Bhargav Teja Nallapu, Nicolas P. Rougier, Bapi Raju Surampudi. The art of scaling up: a computational account on action selection in basal ganglia. 3rd Annual Conference on Cognitive Science (ACCS 2016), Oct 2016, Gandhinagar, India. hal-01354041

\section{HAL Id: hal-01354041 \\ https://hal.science/hal-01354041}

Submitted on 17 Aug 2016

HAL is a multi-disciplinary open access archive for the deposit and dissemination of scientific research documents, whether they are published or not. The documents may come from teaching and research institutions in France or abroad, or from public or private research centers.
L'archive ouverte pluridisciplinaire HAL, est destinée au dépôt et à la diffusion de documents scientifiques de niveau recherche, publiés ou non, émanant des établissements d'enseignement et de recherche français ou étrangers, des laboratoires publics ou privés. 


\title{
The art of scaling up : a computational account on action selection in basal ganglia
}

\author{
Bhargav Teja Nallapu ${ }^{1,2}$, Nicolas P. Rougier ${ }^{2,3,4}$ and Bapi Raju Surampudi ${ }^{1,5}$ \\ ${ }^{1}$ International Institute of Information Technology (I.I.I.T), Hyderabad \\ ${ }^{2}$ INRIA, Bordeaux Sud-Ouest, Talence, France \\ ${ }^{3}$ IMN, CNRS, University of Bordeaux, UMR 5293, IMN, Bordeaux, France \\ ${ }^{4}$ University of Bordeaux, CNRS UMR 5800, Labri, IPB, Talence, France \\ ${ }^{5}$ School of Computer and Information Sciences, University of Hyderabad, India
}

\begin{abstract}
What makes a model 'large scale'? Is it the number of neurons modeled? Or the number of structures modeled in a network? Most of the higher cognitive processes span across co-ordinated activity in different brain areas. However at the same time, the basic information transfer takes place at a single neuron level, together with multiple other neurons. We explore modeling a neural system involving some areas of cortex, the basal ganglia (BG) and thalamus for the process of decision making, using a large-scale neural engineering framework, Nengo. Early results tend to replicate the known neural activity patterns as found in the previous action selection model [2], besides operating with a larger neuronal populations. The power of converting algorithms to efficiently weighed neural networks in Nengo [10,1] is exploited in this work. Crucial aspects in a computational model, like parameter tuning and detailed neural implementations, while moving from a simplistic to large-scale model, are studied.
\end{abstract}

Keywords: neural dynamics, basal ganglia, large scale, nengo, decision making

\section{Introduction}

Action selection is one of the most vital processes for the survival of an organism. The very importance of the process of action selection (and learning from it) in the survival of an organism, pushes for a need of understanding the process from an evolutionary, biological and physiological point of view.

Brain works by running complex dynamics forming complex connections within itself, specific to each behavioral repertoire. It is at the level of few (or many) neurons that the information transfer takes place, which drives all the processes like perception, memory, decision making, language or any other brain process. Ideally, it is that level of detail one wants to model any sub-system of brain or the whole model of brain itself. We start with considering one of our previous works on a computational study of decision making and its dynamics using a thalamo-cortical BG network [8] inspired by [2] and replicated in [11]. 
The emphasis is on modeling these functional networks at a large scale, both in terms of neuron populations as well as multiple brain structures. To achieve this, we use a neural engineering framework(NEF) based graphical and scripting tool called Nengo[10].

\section{Methods}

\subsection{Task}

The task is a probabilistic learning task that is described in [9] and used in [2, 11,8]. Four target shapes are associated with different reward probabilities (see figure 1). Every time any two of the four possible shapes are presented at two random positions (out of the four cardinal positions - up, right, down and left). By the end of trial period, a choice is made and the reward is given according to the reward probability associated with the chosen shape. In a single independent

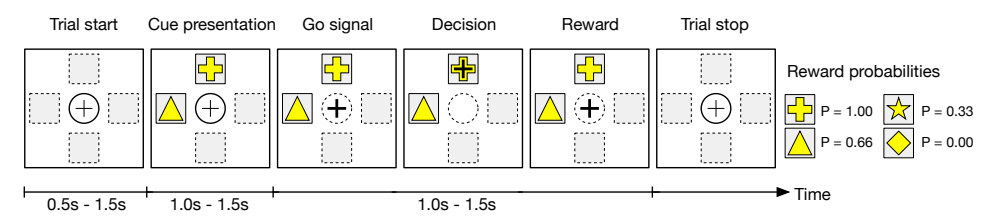

Fig. 1. The two armed bandit task as described in $[9,2]$.

trial, the cognitive decision (shape of the cue) and motor decision (direction of position) are independent of each other. However, ideally, the motor decision is expected to be in the direction of the cue shape that is chosen. On subsequent reward association of the cue decision, one should eventually select the direction in which the most rewarding cue is present.

\subsection{Model}

In [4], authors demonstrated an action selection mechanism in the cortico-basal ganglia loops based on a competition between the positive feedback, direct pathway through the striatum and the negative feedback, hyper-direct pathway through the sub-thalamic nucleus. This model allows a bidirectional information flow between loops such that during early trials, a direction can be selected randomly, irrespective of the cue positions. However, after repeated trials, the model is able to consistently make the motor decision, only after cognitive decision, towards the position of the more rewarding cue shape.

\subsection{The $N E F$ and Nengo}

The underlying principles of NEF are: representation, transformation and dynamics. The activity of a group of neurons is considered to be best represented 

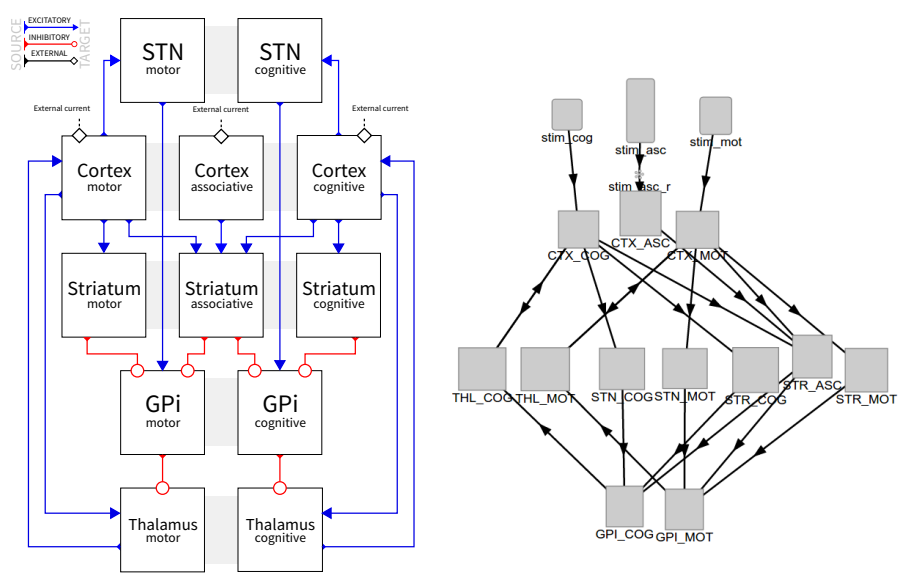

Fig. 2. Architecture of BG model (left) from [2]. A model nengo network (right)

by encoding of some underlying variable, for e.g, any vector generally of smaller dimensionality. The transformation of information from one neural group to the other neural group. i.e., the neural group representing $\mathbf{x}$ can be connected to other group of neurons representing a variable $\mathbf{y}$, such that $\mathbf{y}=f(\mathbf{x})$. Any desired function can be approximated with improved accuracy by increasing the number of neurons. The dynamics of NEF allows us to build recurrent neural networks. These networks can compute the time evolution of a given variable $\mathbf{x}$ of the form $\frac{d \mathbf{x}}{d t}=f(\mathbf{x}, \mathbf{u})$.

\section{Results}

The following simulations assume cue shapes are represented by $[0,1,2,3]$ in the cognitive channel of the cortex and the cardinal directions, where the shapes could be presented, are also represented by $[0,1,2,3]$ in the motor channel of the cortex. The cue shapes have a rewarding probability in the order $1>0>2>3$. The synaptic weights that were learned in the previous model were applied. These weights ensure that the highest rewarding cue is always selected. It is also implicit in the model dynamics that the position corresponding to the chosen shape only will be selected. Figure 3 displays the correct cognitive decision (shape) and corresponding motor decision (position).

\begin{tabular}{lcc} 
Model & Cortical level & Total \\
\hline Simplistic model [2] & 24 & 72 \\
Model implemented & 24000 & 387,200 \\
Full-scale rat model [6] & $180 \mathrm{~K}$ & 3 million \\
\hline
\end{tabular}

Table 1. No. of neurons across models 

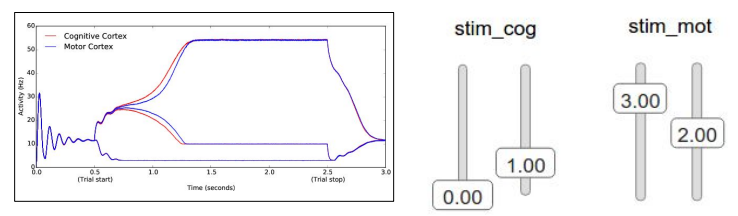

stim_asc
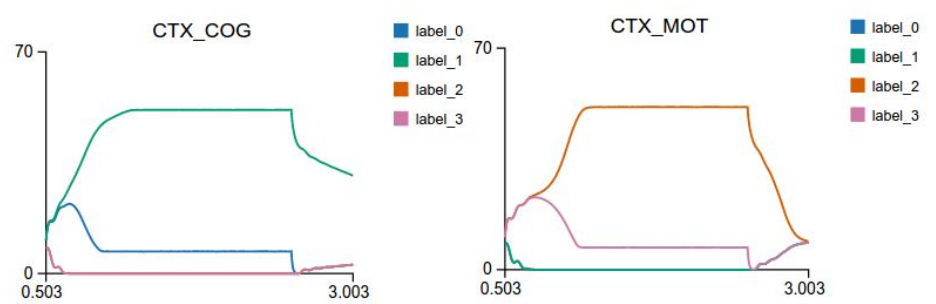

Fig. 3. For the combinations of cue shape and position, the best rewarding cue shape is selected (see CTX_COG) and the corresponding position (as per stim_cog and stim_mot represent shape 0 is shown at position 3 and shape 1 is shown at position 2). It can be seen that, at a point later than choosing shape, corresponding position is chosen (see CTX_MOT). Inset The decision in the original simplistic model

\section{Discussion}

For the models claiming to have network-induced dynamics, the choice of the neuronal dynamics should not make a significant difference. This has been demonstrated clearly in this work. The model developed is tested under similar conditions of action selection task, switching between direct computations and neural implementations like Sigmoidal and Integrate \& Fire (IF). To start with, we used simple neurons that directly compute a function. Then the neurons are changed to detailed implementations, but to a lower scale. However, the switch between neuron implementations involved minimal tuning of parameters of the network as well as optimization of few framework parameters, provided there is computational power to handle the large scale of spiking or IF neurons.

Although the population numbers in this work are comparable to that of experimental numbers of a rat's BG and some recent modeling work by Moren et al. 2015 [6] (see table 1), we are still far away from the scale of that of humans' (150K in rats where as 2.2 million neurons, only the globus pallidus and subthalamic nucleus combined [3]). As for results, the neural activity corresponding to synaptic weight-driven decision making and the ideal choice as per high reward, were consistent. We also believe, at a full range simulations on the model, performance would match that from the previous simplistic model. In future, such a functional large scale network could become readily usable in other functional processes involving motor reaching or motor learning [5], modeling deficits in neuro-degenerative disorders such as Parkinsonianism [7] etc. 


\section{Acknowledgements}

The authors would like to acknowledge the following grants which have been a major support to this research.

- Indo-French CEFIPRA Grant for the project Basal Ganglia at Large (No. DST-INRIA 2013-02/Basal Ganglia dated 13-09-2014)

- Internships programme at INRIA, 6 month Internship with Team Mnemosyne at INRIA Bordeaux - Sud-Ouest

- Travel Support by Nengo $3^{\text {rd }}$ annual Summer School on large-scale brain modeling, University of Waterloo, ON, Canada, $5^{\text {th }}$ June, $2016-17^{\text {th }}$ June, 2016

\section{References}

1. Bekolay, T., Bergstra, J., Hunsberger, E., DeWolf, T., Stewart, T.C., Rasmussen, D., Choo, X., Voelker, A.R., Eliasmith, C.: Nengo: a python tool for building large-scale functional brain models. Frontiers in neuroinformatics 7 (2013)

2. Guthrie, M., Leblois, A., Garenne, A., Boraud, T.: Interaction between cognitive and motor cortico-basal ganglia loops during decision making: a computational study. Journal of neurophysiology 109(12) (2013)

3. Halliday, C.D.H.J.M.H.D.I.F.M.K.H.G.P.G.M.: Comparison of the basal ganglia in rats, marmosets, macaques, baboons, and humans: Volume and neuronal number for the output, internal relay, and striatal modulating nuclei. The Journal of Comparative Neurology 445 (2002), http://gen.lib.rus.ec/scimag/index.php? $\mathrm{s}=10.1002 / \mathrm{cne} .10165$

4. Leblois, A., Boraud, T., Meissner, W., Bergman, H., Hansel, D.: Competition between feedback loops underlies normal and pathological dynamics in the basal ganglia. Journal of Neurosciences 26, 3567-3583 (2006)

5. Magdoom, K., Subramanian, D., Chakravarthy, V.S., Ravindran, B., Amari, S.i., Meenakshisundaram, N.: Modeling basal ganglia for understanding parkinsonian reaching movements. Neural Computation 23(2), 477-516 (2011)

6. Moren, J., Igarashi, J., Yoshimoto, J., Doya, K.: A full rat-scale model of the basal ganglia and thalamocortical network to reproduce parkinsonian tremor. BMC Neuroscience 16(1), 1 (2015)

7. Muralidharan, V., Balasubramani, P.P., Chakravarthy, V.S., Lewis, S.J., Moustafa, A.A.: A computational model of altered gait patterns in parkinsons disease patients negotiating narrow doorways (2015)

8. Nallapu, B.T., Rougier, N.P.: Dynamics of reward based decision making a computational study. In: International Conference on Artificial Neural Networks (2016)

9. Pasquereau, B., Nadjar, A., Arkadir, D., Bezard, E., Goillandeau, M., Bioulac, B., Gross, C.E., Boraud, T.: Shaping of Motor Responses by Incentive Values through the Basal Ganglia. Journal of Neuroscience 27(5) (2007)

10. Stewart, T.C., Tripp, B., Eliasmith, C.: Python scripting in the nengo simulator (2009)

11. Topalidou, M., Rougier, N.: [re] interaction between cognitive and motor corticobasal ganglia loops during decision making: a computational study. ReScience 1(1) (2015) 